\title{
El proceso de lectura en el Sistema de Información Documental
}

\author{
The reading process in documentary information system
}

Miguel Ángel Rendón Rojas

Doutor em Filosofia pela Universidade Estatal de Moscou "M.V. Lomonósov".

Pesquisador do Centro Universitario de Investigaciones Bibliotecológicas da Universidade Nacional Autónoma do

México

E-mail: marr@servidor.unam.mx

Elia Magdalena Martínez Sánchez

Licenciada em Bibliotecología pelo Colegio de Bibliotecología da Facultad de Filosofía y Letras. Pós-graduanda do Programa de Pós-graduação de Lingüística Hispánica da Universidade Nacional Autónoma do

México.

E-mail: simoon97@hotmail.com

\section{Resumen}

Se concibe a la lectura como un proceso y no como el resultado en sí. Se analizan las etapas que constituyen ese proceso desde el punto de vista lingüístico, cognoscitivo y hermenéutico. Partiendo de la distinción entre el lenguaje interno y el lenguaje externo que hacía la filosofía medieval, se toma la información como un ente ideal análogo al lenguaje interno y al documento como su objetivación. En el proceso de la lectura se realiza la desobjetivación de la información.

Palabras Claves: Proceso de lectura, información, documento.

\begin{abstract}
Reading is conceived as a process and not as a result. The reading process stages are analyzed from a linguistic, a cognitive and a hermeneutic points of view. From the perspective of the medieval philosophy a distinction is made between inner and external language, so information is taken as an ideal entity similar to inner language and a document is seen as its objetivation. During the reading process the deobjetivation of information is carried out.
\end{abstract}

Keywords: Process of reading, information, document. 


\section{Introducción}

El fenómeno de la lectura, dada su complejidad, ha sido investigado desde diferentes perspectivas por varias disciplinas, tales como la lingüística, la psicología, la hermenéutica, la sociología y, por supuesto, también la bibliotecología. Una de las principales razones para el estudio de la lectura en bibliotecología es que gran parte de los documentos que integran los acervos de las bibliotecas poseen un texto escrito y el contacto de los usuarios con estos textos sólo puede ser posible a través de la lectura. Entre las líneas de investigación sobre este tema en bibliotecología podemos mencionar el fomento a la lectura, el papel del bibliotecólogo como promotor de la lectura, y la tipificación de lecturas de acuerdo con determinadas épocas o grupos sociales.

Nuestra propuesta en el presente trabajo es analizar la lectura desde el proceso mismo que la constituye, es decir, concebimos a la lectura como un proceso y no como el resultado en sí. La lectura es la actividad de leer y no el acto de haber leído. Así pues, partiendo de ese supuesto, encontramos que dentro del proceso de la lectura intervienen algunos elementos sin los cuales sería imposible la lectura, porque actúan como agentes activos que le dan la existencia misma y simultáneamente posibilitan una serie de estadios, lo que significa que motivan el desarrollo de dicho proceso. Estos elementos son explícitos como el texto y el lector e implícitos como el autor, la cultura y la sociedad. Entre esos componentes existe una tensión dialéctica entre la objetivación y desobjetivación de la información, que es precisamente el motor que hace posible el movimiento de la idea a la escritura, de ésta a la lectura y finalmente de la lectura a la idea nuevamente. Explicar por qué y cómo se da ese movimiento es el objetivo de este artículo.

La importancia de un estudio como el presente es obvia para el profesional de la información documental, puesto que al comprender mejor el fenómeno de la lectura se tendrá al mismo tiempo una base teórica más firme para ofrecer servicios acordes a las necesidades de los usuarios-lectores.

\section{El proceso de lectura}

InCID: R. Ci. Inf. e Doc., Ribeirão Preto, v. 1, n. 2, p. 133-152, jul./dez. 2010. 


\section{Constitución del texto}

El texto está compuesto por una serie de elementos lingüísticos (morfosintácticos, léxicos, semánticos) que se ponen en funcionamiento para construir formas de comunicación y de representación del mundo (CALSAMIGLIA; TUSÓN, 2007, p. 1).

En un nivel morfosintáctico se dan las estructuras de forma, combinación y distribución de palabras y secuencias de oraciones. Dichas secuencias adquieren coherencia cuando a las estructuras sintácticas se les asigna significado en relación con los hechos a los que hacen referencia. En el nivel semántico la atención se centra en el significado, es decir, en la proposición que subyace en una oración. Una proposición hace referencia a una situación determinada de un hecho real o de uno posible.

Para dotar de sentido y coherencia a las proposiciones de un texto se debe establecer una conexión entre los hechos anteriores y los hechos posteriores a los que hacen referencia. Un ejemplo de ello es la función Tema y Rema. El Tema es la información conocida, luego se da la información nueva, es decir, el Rema. La información conocida y la información nueva dan otra información conocida y así sucesivamente. Con ello se establece una especie de relación cíclica que a su vez está estrechamente vinculada con otras conexiones que sólo son posibles si las circunstancias de determinadas situaciones a las que hacen referencia están relacionadas. Las conexiones entre circunstancias pueden darse en forma directa en proposiciones explícitas (lo que está propiamente en el texto) y en forma indirecta en proposiciones implícitas (lo que está en el contexto y en el conocimiento general del mundo).

\section{Las estructuras textuales: micro-, macro- y superestructuras}

Las estructuras textuales son "los procesos o mecanismos que determinan la configuración de un texto" (BERNÁRDEZ, 1987, p. 17). Dichas estructuras se dividen en microestructuras, macroestructuras y superestructuras. Las microestructuras son las oraciones y secuencias de oraciones de un texto, en este nivel se comprende el texto de manera local extrayendo los significados o proposiciones de las oraciones. Las macroestructuras están formadas por macroproposiciones construidas sobre la base de las conexiones entre las 
secuencias completas de oraciones y el conocimiento previo. En este nivel se conoce el tema o contenido global del texto. Las superestructuras son las estructuras narrativas, argumentativas, descriptivas, expositivas, etc., con las que se construyen los diversos textos (novelas, poesías, conferencias académicas, informes, etc.).

Un texto es coherente en la medida en que por medio de la unión de las micro- y macroestructuras se le puede asignar un sentido local y global, y de éstos un tema o asunto general. Las macrorreglas pueden ser de gran ayuda en este proceso ya que su función es obtener el significado global de una serie de proposiciones reduciendo la información semántica. Algunas macrorreglas propuestas por van Dijk $(1983,2001)$ son: omitir (se omiten las proposiciones que el sujeto no considera importantes), generalizar (se generaliza una secuencia de proposiciones por medio de una proposición que señala el tema general de la secuencia) y construir (se suprimen los detalles que pertenecen convencionalmente a una acción y se construye una proposición que designa la acción de manera global).

\section{Constitución del sujeto cognitivo}

Al mismo tiempo en el proceso de lectura tienen lugar, además de procesos lingüísticos, procesos cognoscitivos. Según Neisser (1976, p. 14), “el término "cognición” se refiere a todos los procesos mediante los cuales el ingreso sensorial es transformado, reducido, elaborado, almacenado, recobrado o utilizado". El lugar donde se realizan estos procesos es el sistema cognoscitivo humano, el cual está compuesto básicamente de: “ a) un dispositivo para recibir información acerca del medio ambiente; dicho dispositivo está representado por los receptores, b) una manera de ejecutar acciones sobre el mundo -control motor-, y c) procesos cognoscitivos que incluyen: medios para identificar e interpretar la información recibida por los receptores; medios para controlar las acciones a ejecutar; medios para dirigir la selección de recursos cognoscitivos cuando existe un número de necesidades mayor que las que pueden satisfacer de manera inmediata (recursos finitos); y una memoria para la historia de las experiencias y las acciones" (NAVARRO, 1995, p. 30).

InCID: R. Ci. Inf. e Doc., Ribeirão Preto, v. 1, n. 2, p. 133-152, jul./dez. 2010. 


\section{Sistemas de memoria: memoria sensorial, memoria operativa y memoria semántica}

La estructura de la memoria está compuesta por tres sistemas. La memoria sensorial "es la memoria tipo cámara fotográfica, de una amplia capacidad, pero de muy corto período de duración. Codifica los atributos de los estímulos tal como se presentan en su textura sensorial" (POGGIOLI, 1995, p. 282). La memoria operativa distribuye los recursos cognoscitivos. Según Miller $^{1}$ (cit. NAVARRO, 1995, p. 10), su capacidad oscila entre cinco y nueve unidades de información, ya se trate de números, palabras, etc., con una duración que oscila entre diez y veinte segundos. Por su parte, Baddeley ${ }^{2}$ (cit. POZO, 1999, p. 129) divide esta memoria en tres subsistemas: el lazo articulatorio, que procesa información de naturaleza fonológica, la agenda visoespacial, que procesa información de naturaleza espacial, y el ejecutivo central, que controla el sistema de memoria distribuyendo los recursos cognoscitivos a los otros subsistemas y buscando información en la memoria semántica. La memoria permanente o semántica es un sistema de capacidad y duración ilimitada. Se cree que la información que es almacenada en esta memoria puede durar toda la vida. En la medida en que estos tres sistemas de memoria se organicen y se sincronicen, los procesos cognoscitivos que en ellos se realizan serán más efectivos.

\section{La representación del conocimiento}

Las investigaciones realizadas por Gagné, Nayes-Roth y Thorndyke, entre otros, proponen que la información conceptual está almacenada en la memoria permanente en forma de redes de proposiciones. Una proposición es una forma de representar la información en la memoria, ya que "generalmente recordamos las ideas pero no necesariamente las palabras exactas que se utilizaron para comunicarlas" (GAGNÉ, 1991, p. 82). Las proposiciones en la memoria se representan en forma de nodos y eslabones. "Los nodos, o círculos, representan la totalidad de la proposición. Los eslabones, o flechas, indican cada elemento de la proposición. Los eslabones están etiquetados para que pueda identificarse el papel de cada elemento dentro de una

\footnotetext{
${ }^{1}$ MILLER, George A. The magical number seven, plus or minus two: some limits on our capacity to for processing information. Psychological Review, v. 63, 1956. p. 81-97.

${ }^{2}$ BADDELEY, A. D. Human memory: theory and practice. Psychology Press, 1990.

InCID: R. Ci. Inf. e Doc., Ribeirão Preto, v. 1, n. 2, p. 133-152, jul./dez. 2010.
} 
proposición específica” (GAGNÉ, 1991, p. 84). Las proposiciones que presentan significados comunes están interrelacionadas entre sí, formando redes proposicionales con una organización jerárquica que incluye categorías, principios y generalizaciones. Las proposiciones se activan según se vayan necesitando en determinado momento, y representan una parte de lo que se está pensando. Las proposiciones activadas pasan de la memoria semántica a la memoria operativa donde se unen con nuevas proposiciones. Las proposiciones producto de esta unión son enviadas a la memoria semántica. Esto permite entender mejor los procesos de adquisición y recuperación de la información en la memoria.

La adquisición de la información será más efectiva en la medida en que se logren integrar las proposiciones nuevas con las proposiciones relacionadas almacenadas en la memoria. Esta integración modifica, en mayor o menor grado, la estructura de las proposiciones primigenias que le dieron significado a las proposiciones nuevas. A este proceso se le conoce como construcción de conocimientos.

\section{El proceso de lectura desde un enfoque lingüístico y cognoscitivo}

Para realizar el acto de lectura es necesario un texto bien estructurado, un sujeto cognoscitivo y una serie de estadios intermedios con duración determinada, es decir, un proceso. Dicho proceso posee características invariables, pero a la vez es flexible en cuanto considera las diferencias entre las estructuras de las lenguas, las características de los textos y los propósitos, objetivos e intereses del lector.

\section{Decodificación}

En la decodificación se da el primer contacto con la información del texto. La memoria sensorial capta los primeros estímulos (información morfosintáctica) que son transferidos a la memoria operativa donde el ejecutivo central comienza a buscar y a activar en la memoria semántica las proposiciones relacionadas con las palabras que aparecen en la oración permitiendo darles un significado.

InCID: R. Ci. Inf. e Doc., Ribeirão Preto, v. 1, n. 2, p. 133-152, jul./dez. 2010. 


\section{Comprensión local del texto}

La comprensión de las oraciones y secuencias de oraciones tiene un carácter cíclico, cuyo objetivo es unir la información nueva con la información ya conocida. La mayor parte de este proceso cíclico se realiza en la memoria operativa, que a pesar de tener una capacidad limitada es suficiente para: a) vincular la información de las oraciones, b) integrar secuencias de oraciones en proposiciones, c) seguir leyendo las oraciones consecutivas (información nueva) y vincularlas con las proposiciones anteriores (información vieja), d) unir de forma coherente las proposiciones estableciendo relaciones de conexión: tema y rema, proposiciones explícitas y proposiciones implícitas de la memoria semántica, e) transferir constantemente a la memoria semántica las series de proposiciones, que podrán ser remitidas de nuevo a la memoria operativa en cuanto se necesiten.

Una vez que la información de las microestructuras se ha traducido a información proposicional se borra de la memoria operativa. Por esta razón no se suelen recordar literalmente las oraciones y secuencias de oraciones, aun inmediatamente después de haberlas leído, únicamente se recuerdan las proposiciones formadas de las oraciones que han logrado almacenarse en la memoria semántica.

Las proposiciones explícitas se establecen sobre la base de las oraciones y secuencias de oraciones del texto, las cuales están interconectadas por relaciones gramaticales que guían la cohesión y coherencia interna del texto. La comprensión literal necesita ser complementada con las proposiciones implícitas del contexto situacional y del contexto cultural almacenado en la mente del lector, quien al utilizar su conocimiento previo para comprender, entra en una serie de transacciones con el texto, lo cual hace que su proceso receptivo sea un proceso activo.

\section{Comprensión global del texto}

Este tipo de comprensión está integrada por varios niveles de macroproposiciones organizadas jerárquicamente en una macroestructura más general que representa el tema en cuestión. Las macroestructuras participan inversamente en el proceso de lectura. En primer lugar, permiten la comprensión global al ser el resultado de: a) las oraciones y proposiciones del texto,

InCID: R. Ci. Inf. e Doc., Ribeirão Preto, v. 1, n. 2, p. 133-152, jul./dez. 2010. 
b) las conexiones hechas del conocimiento implícito proveniente del contexto y del conocimiento convencional del mundo, y c) la serie de macroproposiciones construida sobre la base de todos estos factores. En segundo lugar, pueden ayudar al lector a deducir el probable tema del texto que se desea leer y con base en eso establecer suposiciones y expectativas del tema, éstas crean macroproposiciones hipotéticas que influyen en la conexión y comprensión de las oraciones y proposiciones del texto, y pueden ser confirmadas o desechadas en el proceso de lectura.

En este nivel de comprensión las macrorreglas (omitir, generalizar y construir) son consideradas procesos mentales, que ayudan a reducir la información, ya sea suprimiendo detalles o generalizando proposiciones, lo cual asegura la comprensión, organización y almacenamiento de las ideas centrales del texto en la memoria.

Por otra parte, las superestructuras esquemáticas al establecer la estructura y organización del contenido de los tipos de texto, también organizan el proceso de lectura. Cada tipo de texto tiene una estructuración específica que no necesariamente puede ser conocida por el lector. Sin embargo, sí puede saber que cada tipo de texto puede satisfacer determinadas exigencias y objetivos, lo que determina su conveniencia de acuerdo con los intereses de cada lector. Así pues, en mayor o menor grado, las superestructuras predisponen al lector, que al reconocer el tipo de texto que desea leer, espera cierto ordenamiento micro- y macroestructural característico del texto.

Los procesos de comprensión en el nivel de las micro- y macroestructuras se realizan de manera simultánea, ya que a la vez que se comprenden las secuencias de oraciones y proposiciones del texto también se va comprendiendo el texto de forma global. El resultado de ambos tipos de comprensión -que en condiciones óptimas sería la comprensión general del texto, se transfiere a la memoria semántica donde se produce un reacomodo de la red proposicional.

InCID: R. Ci. Inf. e Doc., Ribeirão Preto, v. 1, n. 2, p. 133-152, jul./dez. 2010. 


\section{La comprensión: un enfoque hermenéutico}

El enfoque hermenéutico nos permitirá tener una visión más completa de otros factores que ocurren durante el proceso de lectura y que determinan la comprensión.

El término Hermenéutica "procede del dios "Hermes" - el mensajero -, al que la mitología asigna la tarea de mediador intérprete entre los dioses y los hombres, sirve para expresar el significado de "anunciar" o "transmitir" algo" (MARTÍNEZ, 1994, p. 81).

El autor realiza el proceso de producción del texto, el cual es inverso al proceso que hace el lector cuando lee un texto. Por lo general, un autor tiene como punto de partida una macroestructura (tema o asunto principal) y una superestructura, que lo guían durante la producción de estructuras semánticas y estructuras morfosintácticas de tal forma que se logre un texto coherente. Así pues, "la generación de un texto ha de partir de una idea principal que, gradualmente, se va desarrollando diversificándose en significados cada vez más detallados que van penetrando en el interior de los fragmentos simples de longitud oracional" (BEAUGRANDE; DRESSLER, 1997, p. 65). Es precisamente la transmisión de esta idea o ideas una de las intenciones que lo mueven a producir el texto. Dichas ideas son un reflejo de su entorno histórico, cultural y social; y están expresadas utilizando un lenguaje compartido, que no es exclusivamente suyo, pero que a la vez impregna con su individualidad, con sus experiencias, pensamientos y estilo. Es dentro de este lenguaje común, donde se realiza el entendimiento y la comprensión entre el texto y el lector.

Según Gadamer (1977), para lograr el entendimiento con el texto es necesario pasar por la precomprensión, ésta supone una toma de conciencia respecto de las opiniones y supuestos previos (prejuicios) del lector, en la cual se deben dejar a un lado los prejuicios que en un momento dado pueden limitar o impedir el entendimiento con el texto y sólo se deben mantener aquellos prejuicios que orienten la correcta comprensión. Para poder valorar estos prejuicios es preciso tomar "distancia histórica", lo cual significa considerar las condiciones históricas propias del texto que quiere ser comprendido y del lector que lo quiere comprender. Al estar determinados por estas condiciones, texto y lector tienen sus propios horizontes. Los horizontes les permiten situarse por encima de sus campos de referencia, pero, sin olvidarlos. En la medida en que ambos horizontes se fusionen se realizará una adecuada comprensión. La fusión de

InCID: R. Ci. Inf. e Doc., Ribeirão Preto, v. 1, n. 2, p. 133-152, jul./dez. 2010. 
horizontes sólo es posible entablando un diálogo entre el texto y el lector. En este diálogo debe existir una total igualdad, una desvinculación de los prejuicios negativos y una apertura y reconocimiento hacia el otro. La dialógica gadameriana pretende que ambas partes lleguen a un acuerdo, a una comprensión compartida que da lugar a la ampliación de sus propios horizontes. Así también, pretende alcanzar el punto medio entre objetividad y subjetividad, en donde se encuentra lo objetivo en lo subjetivo, es decir, pretende alcanzar la "subjetualidad".

De esta forma, los factores históricos, culturales y sociales influyen en la interpretación y comprensión de los textos, y éstos a su vez, han ejercido y ejercen su influencia en dichos factores. Esto significa que el todo (cultura, sociedad) se comprende desde lo individual (lector, texto) y lo individual desde el todo. Dentro de este proceso el lector llega a la comprensión de sí mismo y de su entorno.

\section{La información documental como logos ideal}

Una de las tareas a cumplir en la fundamentación de una disciplina es analizar y esclarecer, dentro de su marco teórico, el contenido de los conceptos centrales utilizados en dicha disciplina; esto es, dentro de la fundamentación teórica es necesario definir o redefinir esos conceptos y precisar bajo qué aspecto son enfocados. Entre algunos de los conceptos básicos en la bibliotecología encontramos el de información (RENDÓN, 2005, p. 81). La tarea es delimitar dentro del campo bibliotecológico cuál es el aspecto de información que le interesa. Para ello partiremos de algunas concepciones filosóficas y semióticas que nos proporcionarán los elementos necesarios de ese concepto central.

Nuestra visión tiene sus raíces en las ideas de San Agustín y los escolásticos, quienes plantearon, dentro de la filosofía del lenguaje, la existencia de un lenguaje interno y de un lenguaje externo (BEUCHOT, 1988, p. 27). El primero está constituido por palabras internas o intenciones del alma, es decir, por sentidos, significados, ideas, conceptos. El segundo está integrado por el lenguaje oral y por el lenguaje escrito. Los seres humanos compartimos el mismo lenguaje interno, lo único que varía es el lenguaje externo con el que lo comunicamos. 
Los signos materiales (palabras escritas u orales) no significan directamente a las cosas, sino que lo hacen de manera mediata a través de los signos ideales, es decir, a través de los significados o conceptos, los cuales representan directamente a las cosas designadas.

Siglos después, Gottlob Frege (1998, p. 85), continuando con la tradición medieval, manifestó que las palabras externas poseen Sentido y Referencia. El sentido "es aquello que entendemos al captar una expresión, y es lo que nos sirve de guía para conocer la referencia de la expresión" (BEUCHOT, 1987, p. 74). Lo que entendemos al captar una expresión no es algo subjetivo, sino un contenido objetivo que puede ser captado por muchas personas. Los sentidos pueden ser expresados en diversos lenguajes, lo cual no impide que podamos captar los mismos sentidos, ya que lo único que cambia son los signos externos, no los sentidos. La referencia es aquello que los signos, nombres u expresiones designan.

Por otra parte, Husserl (1985) distinguió entre los signos y las expresiones. Los signos son siempre signos de algo, cumplen una función indicativa o señalativa, pero pueden no expresar nada, a menos que, además de la función indicativa, cumplan con una función significativa. Este hecho los convierte en signos significativos o expresiones.

En toda expresión se dice algo y eso que dice es su significación. La significación es concebida como una unidad ideal-objetiva, que al no depender de circunstancias subjetivas hace que la significación de una expresión sea siempre idéntica. Las significaciones o unidades ideales son conceptos, juicios y raciocinios.

De acuerdo con los planteamientos anteriores, denominamos logos a las entidades ideales, es decir, palabras internas o intenciones del alma, sentidos y significaciones (significados). Dichas entidades ideales no son algo subjetivo; no obstante, sí dependen del sujeto en cuanto que necesitan de éste para existir. El logos no es algo que exista por sí mismo, no se trata de un mundo de las ideas con existencia propia al estilo platónico, sino que existe como contenido intelectual de los sujetos. ${ }^{3}$

El logos, por su estado ideal, no puede ser captado directamente por los sentidos, por ello se necesita de algo material para poder captarlo, es decir, de los signos externos que pueden ser de diferente naturaleza. Así, cuando queremos comunicar nuestro lenguaje interno, éste pasa de

\footnotetext{
${ }^{3}$ Para ampliar nuestra idea de la objetividad del logos sin caer en el idealismo objetivo $c f$. Rendón Rojas, 2005.
}

InCID: R. Ci. Inf. e Doc., Ribeirão Preto, v. 1, n. 2, p. 133-152, jul./dez. 2010. 
un estado ideal a un estado material, de la palabra interna a la palabra externa. Cuando comunicamos las palabras internas utilizando un lenguaje escrito, plasmamos el logos en un objeto, lo materializamos.

Dentro de los documentos que le interesan a la bibliotecología se encuentran aquellos que poseen una unidad comunicativa, es decir, un texto. Como ya se ha visto, el texto está constituido

por un lenguaje escrito que se apega a ciertas estructuras textuales, por lo tanto, se puede decir que el texto es el logos objetivado en un documento.

La información semántica tiene un carácter ideal, en ella encontramos conceptos, significaciones y sentidos, por ello se representa utilizando diversos lenguajes externos. Cuando el autor realiza el proceso de producción del texto, cuando objetiva el logos, lo hace con base en dicha información, esto hace posible la construcción de oraciones inteligibles.

La información que le interesa a la bibliotecología es esa información que se objetiva en los documentos; cada uno de los documentos que integran las colecciones de las bibliotecas es una objetivación del logos; cada uno de estos documentos fue creado con la intención de comunicar una idea, un conocimiento, un determinado mundo de la información y, por lo tanto, es este tipo de información documental la que el usuario intenta buscar y encontrar cuando se dirige a una biblioteca.

\section{El proceso de lectura en el Sistema de Información Documental}

El Sistema de Información Documental (SID) está constituido por el usuario, el bibliotecólogo, la biblioteca, el documento y la información. El SID es un sistema dinámico donde sus elementos subjetivos (usuario y bibliotecólogo) llevan a cabo acciones y actividades, y donde también ocurren sucesos y procesos.

Las interrelaciones en el SID son motivadas por un primer agente (el usuario) cuya estructura cognoscitiva y factores externos determinan el surgimiento de ciertos estados en los cuales necesita información documental (RENDÓN, 2005, p. 111-114). Tales estados pueden considerarse como un estado inicial. El sujeto quiere cambiar ese estado inicial de necesidad por un estado final de satisfacción, para lo cual emprende una acción. De acuerdo con van Dijk 
(1983, p. 85), cuando llevamos a cabo una acción tenemos la intención y el propósito de ejecutar un hacer. Dicho de otra manera, "la mayoría de las acciones son ejecutadas intencionalmente para realizar o producir alguna otra cosa, esto es, otras acciones, sucesos, situaciones o estados mentales; es decir, las acciones tienen metas y esto hace que sean significativas o tengan un "sentido", lo que a su vez hace que sus actores parezcan tener algún propósito" (VAN DIJK, 2000, p. 28). En este sentido, dirigirse a una biblioteca es una primera acción que realiza el sujeto con la intención de satisfacer sus necesidades de información (producir el estado final) y con el propósito de hacer algo, por ejemplo: un trabajo escolar, una investigación, recrearse, etc., de acuerdo con su proyecto de vida. ${ }^{4}$ Esto implica que se debe distinguir entre intenciones y propósitos. De tal forma que cada sujeto puede ir a la biblioteca con la intención de satisfacer sus necesidades de información, pero con propósitos distintos. Ahora bien, cuando este sujeto cognoscitivo entra en contacto con la biblioteca y hace uso de los servicios, acervo, información, instalaciones, etc., que ahí se proporcionan, se convierte en un usuario. El usuario es considerado la razón de ser del Sistema de Información Documental, pero al ser él también un elemento del SID, está en interdependencia con los otros elementos que proporcionan las condiciones para que pueda satisfacer sus necesidades de información.

Anteriormente se mencionó que el tipo de información que el usuario necesita y busca es la información objetivada en los documentos. Estos documentos al haber sido creados con la intención de transmitir información y conocimiento, son unidades comunicativas que poseen el logos objetivado de diversos autores y, con ello, también poseen una parte de la ideología, de la cultura y de la sociedad de la época en la que fueron creados. De ahí que se considere a la biblioteca como uno de los espacios de la memoria social; es decir, la biblioteca al tener entre sus funciones la conservación de los documentos, conserva la memoria social. Pero no sólo la conserva, sino que también propicia su comunicación, permitiendo así que el documento tenga una vida significativa. Desde esta perspectiva, usuario y documento se necesitan mutuamente: el primero para satisfacer sus necesidades de información, el segundo para cumplir su cometido.

\footnotetext{
${ }^{4}$ Hay que puntualizar que la acción de dirigirse a la biblioteca es una acción que a su vez se compone de varias acciones; pero en general, en la percepción e interpretación es vista como una sola acción. Lo mismo sucede con las acciones que el sujeto realiza para satisfacer sus necesidades de información una vez que se encuentra en la biblioteca.
}

InCID: R. Ci. Inf. e Doc., Ribeirão Preto, v. 1, n. 2, p. 133-152, jul./dez. 2010. 
La biblioteca se presenta de esta forma como la institución social que, entre otras cosas, proporciona las condiciones que hacen posible el encuentro del documento con el usuario y con ello se dé paso al proceso de lectura. Algunas de estas condiciones son: el desarrollo, organización y conservación de las colecciones documentales, ${ }^{5}$ los servicios bibliotecarios, los espacios de lectura y mobiliario adecuado, etcétera.

Cuando el usuario ha encontrado el documento, lo abre para encontrarse con el texto y comenzar así el proceso de lectura. Con ello el usuario se convierte en un lector, esto es, si en un primer momento el sujeto cognoscitivo pasó a ser un usuario de la biblioteca, en cuanto se encuentra con el documento y comienza el proceso de lectura, pasa a ser un lector, o mejor dicho, un usuario-lector. Podemos considerar el primer encuentro (el del usuario con el documento) como un primer acceso a la información documental: la biblioteca brinda el acceso a las colecciones documentales que posee; y el segundo (el del usuario-lector con el documento-texto) como un acceso directo a la información objetivada: realizando el proceso de lectura se desobjetiva la información y por tanto se accede a ella (RENDÓN, 1998, p. 247). Como puede verse, en ambos casos el campo bibliotecológico hace posible el acceso a la información.

Anteriormente se mencionó que Gadamer considera el fenómeno hermenéutico (la interpretación y comprensión de los textos) como un diálogo que tiene lugar entre dos personas, además sostiene que entre ambas situaciones en apariencia tan disímiles se establece cierta analogía, a saber, "que toda comprensión y todo acuerdo tienen presente alguna cosa que uno tiene ante sí. Igual que uno se pone de acuerdo con su interlocutor sobre una cosa, también el intérprete comprende la cosa que le dice su texto. [...] la realización de la comprensión, ya se trate de textos o de interlocutores que le presentan a uno el tema, consiste justamente en este llegar a hablar la cosa misma" (GADAMER, 1977, p. 457). Es cierto que el texto no habla propiamente como lo hace un tú, es un objeto y no un sujeto, pero es posible concebir el fenómeno hermenéutico como un diálogo en cuanto que el texto sí llega a hablar, sólo que lo hace a través del lector, esto es, "por él se reconvierten los signos escritos de nuevo en sentido" (GADAMER, 1977, p. 466).

\footnotetext{
${ }^{5}$ Aunque la biblioteca puede poseer varios tipos de materiales (fotografías, mapas, etc.), recuérdese que en este trabajo sólo se consideran aquellos que poseen un texto escrito.

InCID: R. Ci. Inf. e Doc., Ribeirão Preto, v. 1, n. 2, p. 133-152, jul./dez. 2010.
} 
La participación de cada lector en la desobjetivación del sentido hace que el texto se someta a interpretaciones en las que se fusionan la repetición de lo fijado en el texto y la propia innovación que aporta el lector. "El paralelismo con la interpretación musical, explica bien esta acepción. El intérprete da vida a una partitura que, sin su tarea, sería tan sólo un papel con trazos pero nunca una sucesión sonora y melódica de sonidos. El intérprete crea y no sólo porque interpreta sino porque él dará una versión que será la suya, como suya es también la personalidad que da el director a la obra" (AGIS VILLAVERDE, 1995, p. 104). La comprensión implica un reajuste continuo entre lo que el texto le ofrece al lector y lo que éste le aporta. A esto se le conoce propiamente como juego hermenéutico.

Asimismo, este modelo dialógico le permite al lector desobjetivar la información del texto reconociendo no sólo las estructuras sintácticas y semánticas sino también las pragmáticas, es decir, las intenciones del autor, así como ciertos rasgos del contexto histórico del texto, que son fundamentales para una correcta interpretación y comprensión. Con esto planteamos acercarnos a las intenciones del autor desde el sentido lingüístico, reconociendo la información pragmática del texto, y no desde el sentido psicológico que intenta reconstruir la personalidad y las motivaciones creadoras del autor. Nuestra pretensión en este trabajo es, por así decir, más sencilla, entendiendo por correcta comprensión la que se realiza sin transgredir o tergiversar el sentido de las palabras del autor. Con ello no se niega el papel activo del lector y sus necesarias e inevitables aportaciones en el proceso de lectura, únicamente decimos que éstas deben partir de la base textual.

Cuando se logra la fusión de horizontes el lector llega a un entendimiento con el texto y el autor. Pero este entendimiento no significa que el lector acepte por completo lo que se dice en el texto, sino que comprende lo que el autor le comunicó, porque estuvo abierto a lo que le quería decir, sin prejuicios negativos de por medio. "El entendimiento consiste no tanto en compartir una idea, como en aceptar que, además de la propia, existen -y coexisten- otras" (FERRER, 1997, p. 43).

La tensión dialéctica que se produce cuando el usuario va desobjetivando la información objetivada en los documentos, da lugar a un movimiento que comienza cuando el autor produce un texto materializando sus ideas, las ideas objetivadas sólo pueden volver a su estado ideal por medio del proceso de lectura. Cuando el usuario-lector convierte el logos objetivado en logos 
ideal, añade nueva información a sus estructuras mentales produciéndose una modificación en sus redes proposicionales: ahora sabe lo que el autor quiso comunicar con su texto. En este sentido, se considera que este acto comunicativo ha sido satisfactorio si el usuario ha logrado añadir a sus redes proposicionales la información que necesitaba. Este movimiento se puede representar en la siguiente figura:

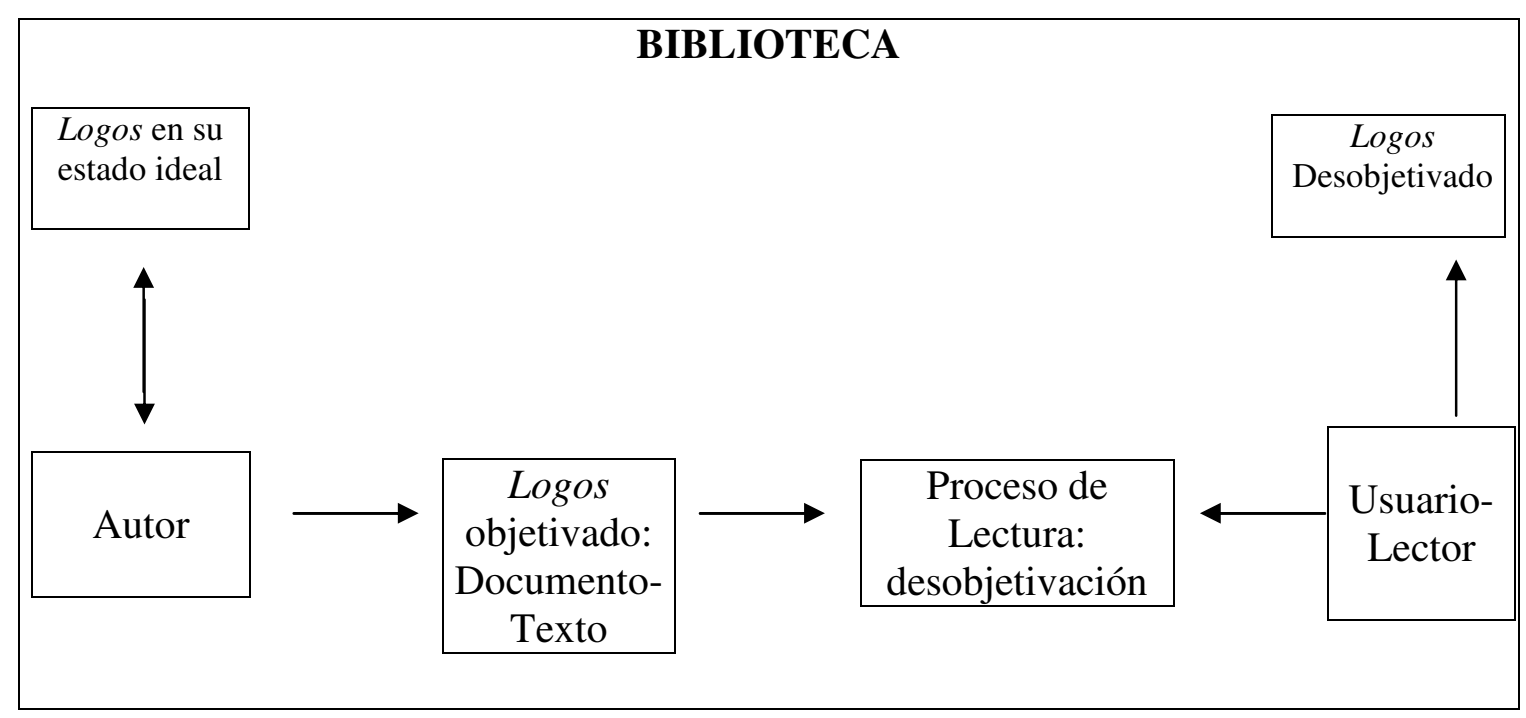

FIG. 1- La desobjetivación de la información a través del proceso de lectura

Así pues, el usuario al dirigirse a la biblioteca se encontraba en un estado inicial de necesidad de información, pero al encontrarse con el documento y realizar el proceso de lectura, donde desobjetiva la información, pasa de ese estado inicial a un estado final de satisfacción de esas necesidades. Esto se puede representar en la siguiente figura:

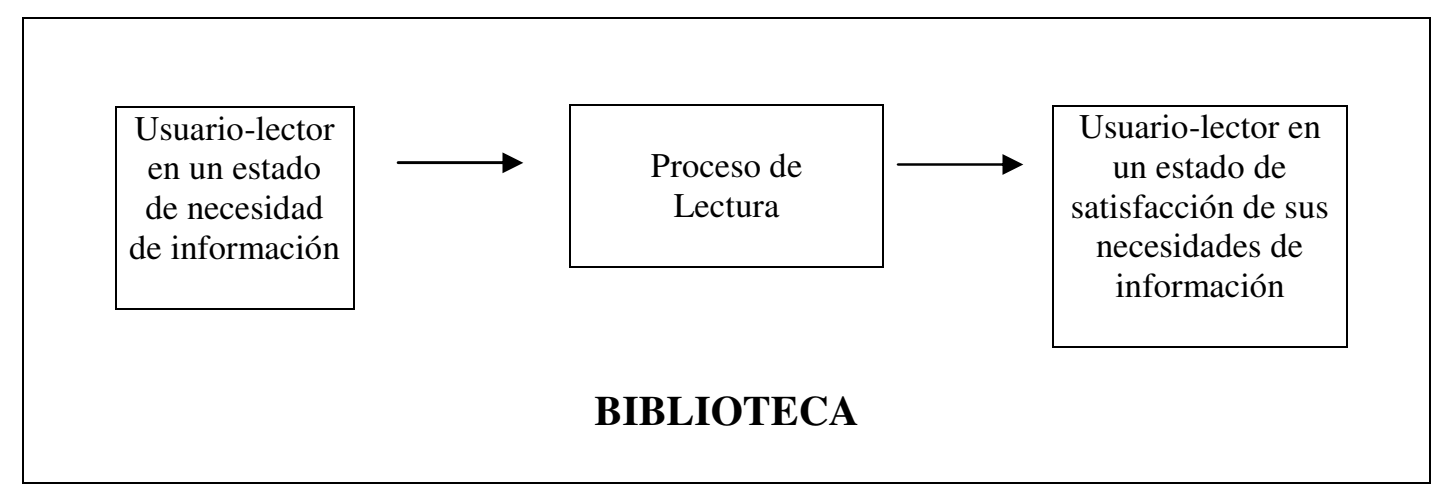

FIG. 2 - La satisfacción de las necesidades de información a través del proceso de lectura 
De acuerdo con la anterior distinción entre intenciones y propósitos podemos distinguir entre satisfactoriedad en la intención y satisfactoriedad en el propósito (VAN DIJK, 1988, p. 252). Así, un usuario pudo haber satisfecho sus necesidades de información, pero no haber realizado el propósito pretendido (no se realizó el trabajo escolar o la investigación, etc.). En este caso hubo satisfactoriedad en la intención, pero no satisfactoriedad en el propósito. Es el usuariolector quien decide, en la medida de sus capacidades y posibilidades, el grado de satisfacción logrado.

Los usuarios usan la información como miembros de una sociedad concreta en la que desarrollan su proyecto de vida. La información, al tener su origen en el mundo objetivo común, les permite entender y cambiar el mundo real donde habitan. Por ello es importante desobjetivar correctamente el logos del autor, porque si la información nos ayuda a entender nuestro entorno, al no llegar a comprender el texto, podemos caer en una red de malentendidos y distorsiones no sólo de las palabras del autor sino también de la realidad.

\section{Conclusiones}

Podemos concluir que la lectura puede definirse como un proceso que tiene lugar debido a la tensión entre la objetivación y desobjetivación de la información. En la objetivación de la información se realiza el movimiento de la idea a la escritura; y en la desobjetivación se pasa de la escritura a la información ideal a través de la lectura.

De acuerdo con su modo de ser, la información ontológicamente aparece como serauténtico o como ser-en-otro. La información ideal se encuentra en su ser-auténtico, mientras que en el documento la información se encuentra en su ser-en-otro. Al realizarse el proceso de lectura la información en su ser-en-otro retorna a la información en su ser-auténtico, es decir, a la información en su estado ideal, por lo que al finalizar el ciclo, la información ya no se encuentra sólo en la mente del autor, ni en el documento sino también en la mente del que leyó el texto.

Con la concepción del documento como la objetivación del logos podemos identificar dos aspectos que se conjugan en el documento: un aspecto ideal y un aspecto físico. El aspecto ideal es el logos del autor que, no obstante la objetivación, conserva su unidad, atemporalidad y objetividad. El aspecto físico es la materia como soporte y como conjunto de signos sensibles, 
cuyas características esenciales son: la temporalidad, el movimiento y el espacio. Lo anterior tiene consecuencias para otras áreas de interés bibliotecológico como lo es el estudio del patrimonio documental, ya que desde esta perspectiva la salvaguarda de dicho patrimonio incluye esos dos aspectos.

El enfoque interdisciplinario de esta investigación nos permite llegar a la conclusión de que el logos o lenguaje interno formado de conceptos, significaciones y sentidos, es la información semántica, que por su naturaleza ideal no puede ser captada directamente por los sentidos, por ello necesita objetivarse utilizando un lenguaje escrito con el cual podemos producir un texto.

El texto está constituido por la información objetivada, por ello es también la objetivación del logos. Esta información del texto que se encuentra en un documento es la información que se maneja en la bibliotecología, es la información que satisface las necesidades de información de los usuarios.

La labor bibliotecológica es fundamental para que se realice el encuentro entre el usuario de la biblioteca y el documento. Este encuentro es una condición necesaria para que el usuario pueda satisfacer sus necesidades de información.

Una vez que el usuario ha vuelto el logos a su estado ideal y lo ha integrado en sus estructuras cognoscitivas, podrá utilizar la información para la realización de su propósito. Cada propósito forma parte del proyecto de vida del usuario, por lo que cada vez que la institución bibliotecaria logra proporcionarle los documentos y la información que necesita para la realización de esos propósitos, contribuye en el desarrollo de su proyecto existencial, así también, al darse esta situación con cada uno de los usuarios, o al menos con la mayoría, contribuye con el desarrollo de la sociedad a la que pertenece.

InCID: R. Ci. Inf. e Doc., Ribeirão Preto, v. 1, n. 2, p. 133-152, jul./dez. 2010. 


\section{Referencias}

AGIS VILLAVERDE, M. Del símbolo a la metáfora: introducción a la filosofía hermenéutica de Paul Ricoeur. [Santiago de Compostela]: Universidad de Santiago de Compostela, 1995.

BEAUGRANDE, R. A.; DRESSLER, W. U. Introducción a la lingüística del texto. Barcelona: Ariel, 1997.

BERNÁRDEZ, E. Introducción. In: BERNÁRDEZ, E. (Comp.). Lingüística del texto. Madrid: Arco/libros, 1987. p. 7-18.

BEUCHOT, M. Aspectos históricos de la semiótica y la filosofía del lenguaje. México: UNAM, Instituto de Investigaciones Filológicas, 1987.

. Significado y discurso: la filosofía del lenguaje en algunos escolásticos españoles postmedievales. México: UNAM, Instituto de Investigaciones Filosóficas, 1988.

CALSAMIGLIA, H.; TUSÓN, A. Las cosas del decir: manual de análisis del discurso, 2. ed. Barcelona: Ariel, 2007.

FERRER, E. Información y comunicación, México: Fondo de Cultura Económica, 1997.

FREGE, G. Sobre sentido y referencia. In: Ensayos de semántica y filosofía de la lógica. Madrid: Tecnos, 1998. p. 84-111.

GADAMER, H. G. Verdad y método: fundamentos de una hermenéutica filosófica. 5. ed. Salamanca: Sígueme, 1977. v. 1.

GAGNÉ, E. D. La psicología cognitiva del aprendizaje escolar. Madrid: Visor, 1991.

HUSSERL, E. Investigaciones lógicas. Madrid: Alianza, 1985. v. 1.

MARTÍNEZ, J. M. La filosofía de las ciencias humanas y sociales de H. G. Gadamer. Barcelona: PPU, 1994.

NAVARRO, A. Psicología cognoscitiva: raíces, supuestos y proposiciones. In: PUENTE, A.; POGGIOLI, L.; NAVARRO, A. Psicología cognoscitiva: desarrollo y perspectivas. Caracas: McGraw-Hill, 1995. p. 1-55.

NEISSER, U. Psicología cognoscitiva, México: Trillas, 1976.

POGGIOLI, L. Estrategias cognoscitivas: una revisión teórica y empírica. In: PUENTE, A.; POGGIOLI, L.; NAVARRO, A. Psicología cognoscitiva: desarrollo y perspectivas, Caracas: McGraw-Hill, 1995. p. 277-322. 
POZO MUNICIO, J. I. Aprendices y maestros: la nueva cultura del aprendizaje. Madrid: Alianza, 1999.

RENDÓN ROJAS, M. A. Bases teóricas y filosóficas de la bibliotecología, México: UNAM, Centro Universitario de Investigaciones Bibliotecológicas, 2005.

. El papel del profesional de la información en el acceso y uso de la información documental. In: La información en el inicio de la era electrónica. México: UNAM, Centro Universitario de Investigaciones Bibliotecológicas, 1998. v. 2. p. 242- 272.

VAN DIJK, T. A. Estructuras y funciones del discurso: una introducción interdisciplinaria a la lingüística del texto y a los estudios del discurso. 13. ed. México: Siglo Veintiuno, 2001.

El discurso como interacción en la sociedad. In: VAN DIJK, T. A. (Comp.). El discurso como interacción social: estudios del discurso: introducción multidisciplinaria. Barcelona: Gedisa, 2000.

. Texto y contexto: semántica y pragmática del discurso. 3. ed. Madrid: Cátedra, 1988.

. La ciencia del texto: un enfoque interdisciplinario. Barcelona: Paidós, 1983.

Artigo submetido em 22 nov. 2010

Artigo aceito em 15 dez. 2010

InCID: R. Ci. Inf. e Doc., Ribeirão Preto, v. 1, n. 2, p. 133-152, jul./dez. 2010. 